\title{
Automated and Objective Numerical Aberration Correction of HRTEM Complex Exit Waves of Crystal Lattices
}

\author{
Colin Ophus, ${ }^{1}$ Martin Linck ${ }^{2}$ and Jim Ciston ${ }^{1}$ \\ ${ }^{1}$ National Center for Electron Microscopy, Lawrence Berkeley National Laboratory, CA, USA \\ ${ }^{2}$ Corrected Electron Optical Systems GmbH, Heidelberg, Germany
}

Hardware aberration correction is a key technology enabling the current generation of high-resolution transmission electron microscopes (HRTEM) to achieve interpretable resolution as low as 50 picometers $[1,2]$. These correctors function by measuring the magnitude and direction of aberrations using a Zemblin tableau, and then using a system of multipole electron lenses that can compensate for the aberrations [3]. This method is effective, but requires a thin, amorphous sample. However, many samples of interest in TEM studies are partially or completely crystalline. After hardware aberration correction, the microscope operator must move to the region-of-interest and then collect data. During this time, the aberration correctors may drift due to electronic instabilities [4], and therefore residual aberrations are common in phase contrast TEM.

One solution to this problem is to numerically correct residual aberrations in the micrograph. If the phase of the electron exit wave can be recovered, for example from a phase plate, inline or offaxis holography, then arbitrary wave aberrations can be added to the micrograph [5]. However the problem then becomes how to measure residual aberrations. When the field of view contains a thin, amorphous object, then we can fit Thon rings to solve residual aberrations. If the sample is thick or crystalline however, the microscopist must manually estimate the aberrations using experience to gauge when the exit wave is aberration-free. This method is time-consuming and prone to error.

In this study, we demonstrate an automatic and objective method to measure and correct undesirable aberrations in HRTEM complex exit waves for crystalline samples. If the sample is made up a series of atomic columns (low index zone axis micrograph of a crystal lattice), we can use a simple figure of merit that reaches a maximum when non-axiosymmetric wave aberrations are zero. As the aberration strength increases, this figure of merit decreases quadratically and monotonically. Figure 1(a) shows wave aberrations applied to a 2D Gaussian weak phase object. Figure 1(b) shows the quadratic decrease of our figure of merit for the aberrations shown in Figure 1(a). By using a gradient search method to maximize this figure of merit as we vary the aberration coefficients, we can measure and correct residual aberrations with no user input. We use simulated complex exit waves with randomly added aberrations to test the accuracy and robustness of our method. We also test the effects of adding noise and sample mistilt to the automated aberration correction routine. Finally, we apply this technique to experimental exit waves reconstructed from both inline and off-axis holography in HRTEM. Figure 2 shows automatic aberration correction applied to experimental exit waves [6]. 


\section{References}

[1] DJ Smith, I Arslan, A Bleloch, EA Stach, ND Browning, PE Batson, PE Batson, N Dellby, OL Krivanek, DA Blom, et al. Development of aberration-corrected electron microscopy. Microscopy and Microanalysis, 14(1):2-15, 2008.

[2] U Dahmen, R Erni, V Radmilovic, C Ksielowski, M D Rossell, and P Denes. Background, status and future of the transmission electron aberration-corrected microscope project. Philosophical Transactions of the Royal Society A: Physical, Mathematical and Engineering Sciences, 367(1903):3795, 2009.

[3] F Zemlin, K Weiss, P Schiske, W Kunath, and KH Herrmann. Coma-free alignment of high resolution electron microscopes with the aid of optical diffractograms. Ultramicroscopy, 3:49-60, 1978.

[4] SM Schramm, SJ Van der Molen, and RM Tromp. Intrinsic instability of aberration-corrected electron microscopes. Physical Review Letters, 109:163901, 2012.

[5] M Linck, B Freitag, S Kujawa, M Lehmann, and T Niermann. State of the art in atomic resolution off-axis electron holography. Ultramicroscopy, 2012.

[6] This work was supported by the Office of Basic Energy Sciences of the U.S. Department of Energy under Contract No. DE- AC0205CH11231.
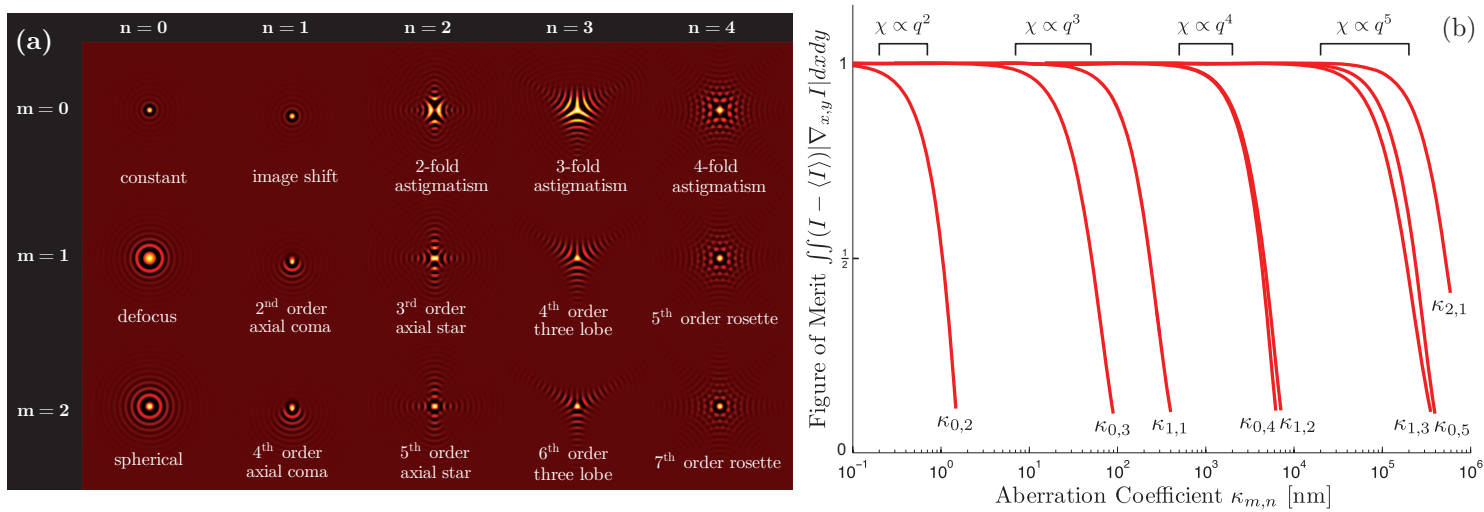

Figure 1: (a) Intensity of a gaussian phase object with $\sigma=0.25 \AA$, over-focused to a white-atom contrast condition, subject to various wave aberrations at $300 \mathrm{kV}(\lambda=1.97 \mathrm{pm})$. The figure of merit defined in this study measured from varying the strengths of the aberrations shown in (a).
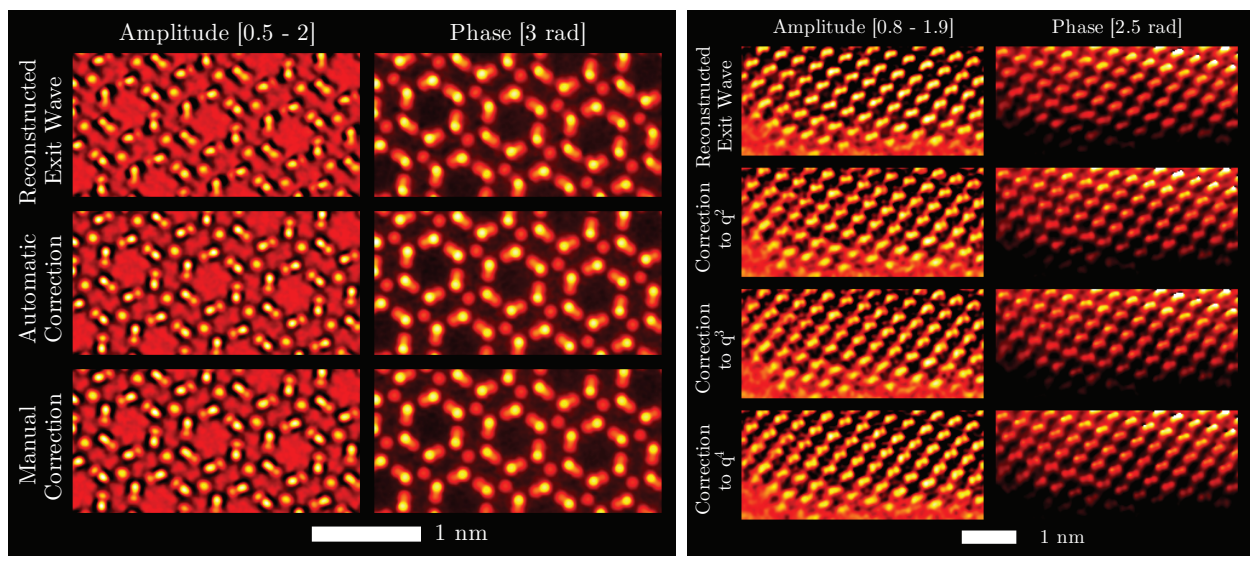

Figure 2: (left) Automated and manual aberration correction for a reconstructed of $\mathrm{Si}_{3} \mathrm{~N}_{4}$ exit wave. (right) Aberrations corrected to varying orders for an off-axis hologram of a Si wedge. 